\title{
Kirsten rat sarcoma (KRAS) oncogene mutation predicts magnitude of response and outcomes in hepatic arterial infusion pump therapy of unresectable colorectal liver metastases
}

\author{
Hordur M. Kolbeinsson ${ }^{1,2} \wedge$, Randa Preihs ${ }^{3}$, Alexandra Bengel ${ }^{3}$, Sreenivasa Chandana ${ }^{4}$, M. Mura Assifi ${ }^{1,2,3}$, \\ Mathew H. Chung ${ }^{1,2,3}$, G. Paul Wright ${ }^{1,2,3}$ \\ ${ }^{1}$ Spectrum Health General Surgery Residency, Grand Rapids, MI, USA; ${ }^{2}$ Department of Surgery, Michigan State University College of Human \\ Medicine, Grand Rapids, MI, USA; ${ }^{3}$ Division of Surgical Oncology, Spectrum Health Medical Group, Grand Rapids, MI, USA; ${ }^{4}$ Cancer and \\ Hematology Centers of West Michigan, Grand Rapids, MI, USA \\ Contributions: (I) Conception and design: All authors; (II) Administrative support: GP Wright; (III) Provision of study materials or patients: R Preihs, \\ A Bengel, GP Wright; (IV) Collection and assembly of data: HM Kolbeinsson; (V) Data analysis and interpretation: HM Kolbeinsson, GP Wright, \\ S Chandana, MH Chung, MM Assifi; (VI) Manuscript writing: All authors; (VII) Final approval of manuscript: All authors. \\ Correspondence to: Hordur M. Kolbeinsson, MD. Department of Surgery, Michigan State University College of Human Medicine, Grand Rapids, \\ MI 49503, USA. Email: hordur.kolbeinsson@spectrumhealth.org.
}

Background: The Kirsten rat sarcoma (KRAS) mutation predicts negative outcomes following resection of colorectal liver metastases (CRLM) and adjuvant hepatic arterial infusion (HAI) pump chemotherapy. Less is known on the effects of KRAS mutation on tumor response in patients with unresectable CRLM undergoing HAI chemotherapy with floxuridine.

Methods: This is a retrospective cohort study investigating the effects of KRAS mutation on tumor response in patients with unresectable CRLM treated with HAI chemotherapy. Primary endpoint was objective response rate (ORR), secondary endpoints included overall tumor response and conversion to resectability.

Results: Twenty-five patients with unresectable liver metastases from colorectal cancer were treated with HAI chemotherapy between 2017-2019. Median number of liver lesions was 12 (range, 1-59) and almost all $(\mathrm{n}=24)$ had prior chemotherapy before starting HAI therapy. Median number of cycles administered via HAI pump was 6 (range, 3-12). Overall decrease in liver tumor burden was 63.5\% (median; range, -257-100\%) with an ORR of 20/25 (80\%) and 10 (40\%) patients converting to resectable status. Eleven (44\%) patients had KRAS positive tumors. When compared to wild-type, KRAS positive tumors had less overall percent decrease $(58 \%$ vs. $70 \% ; \mathrm{P}=0.04)$ and ORR (7/11 vs. 13/13; $\mathrm{P}=0.03)$. Fewer patients with KRAS positive tumors converted to resectable status during HAI therapy (2/11 vs. 8/13; $\mathrm{P}=0.05)$. At a median follow-up of 14.6 months (range, 4.0-36.6 months), overall survival is 45\% among KRAS-positive and 77\% for wild type patients.

Conclusions: KRAS mutational status in patients with unresectable liver metastases from colorectal cancer predicts worse response to HAI chemotherapy compared to wild type.

Keywords: Retrospective studies; colorectal neoplasms; mutation; tumor burden; liver neoplasms

Submitted Aug 18, 2021. Accepted for publication Nov 24, 2021.

doi: 10.21037/jgo-21-514

View this article at: https://dx.doi.org/10.21037/jgo-21-514

^ ORCID: 0000-0002-9076-9280. 


\section{Introduction}

Colorectal cancer is the third leading cause for cancer related deaths in the United States today (1). The high mortality rate is largely due to the development of liver metastases that occurs in approximately $30 \%$ of all patients with colorectal cancer (2). In select cases potential cure can still be reached but only with complete resection of liver metastases and aggressive systemic therapy (3). However, majority of patients with colorectal liver metastases (CRLM) present with unresectable disease $(4,5)$. Utilizing modern systemic chemotherapy with or without targeted therapy, $15-30 \%$ of those patients will respond to a degree that enables resection and a considerable survival benefit $(4,6)$.

Since its introduction in the 1960s, hepatic arterial infusion (HAI) chemotherapy has been predominantly used at select centers as an adjuvant therapy modality for CRLM (7). Despite advances in modern chemotherapy and a decrease in utilization of HAI therapy, recent evidence has shown superior survival data with HAI therapy compared with chemotherapy alone in patients with CRLM (8). These results have been demonstrated both as adjuvant therapy in patients after complete resection and in those with unresectable liver metastases (8-10). Additionally, in the case of unresectable disease, conversion to resectability has been described in up to half of all patients undergoing combination HAI and systemic chemotherapy (11).

The Kirsten rat sarcoma (KRAS) oncogene mutation has been associated with earlier systemic progression of colorectal cancer along with worse outcomes in patients with metastatic disease $(12,13)$. A recent study from Memorial Sloan Kettering Cancer Center on patients undergoing liver resection and adjuvant HAI therapy for CRLM showed patients with KRAS mutated tumors (KRAS-mut) had worse survival compared to wild-type (median overall survival: 67 vs. 47 months, respectively) (14). However, the effect of KRAS mutational status on those treated with HAI chemotherapy for unresectable CRLM has not been a major focus of contemporary studies. This has important clinical applications when considering whether mutational status should be considered before offering HAI chemotherapy for patients with unresectable CRLM. The aim of this study is to examine the impact of KRAS mutational status on tumor response rates and outcomes in a cohort of patients with unresectable CRLM, treated with HAI chemotherapy. We hypothesize that patients with a KRAS mutation will have a lower rate of conversion to resectability compared to wildtype when treated with HAI chemotherapy for unresectable
CRLM. We present the following article in accordance with the REMARK reporting checklist (available at https://jgo. amegroups.com/article/view/10.21037/jgo-21-514/rc).

\section{Methods}

This is a single institution retrospective cohort study of all patients that underwent HAI therapy for unresectable CRLM during the time-period of August 1st, $2017-$ September 1st, 2020. This study was reviewed and approved by the Spectrum Health institutional review board with a waiver of informed consent due to the retrospective analysis of the study (IRB00000883, study ID: 2020-314). The study was conducted in accordance with the Declaration of Helsinki (as revised in 2013). Study inclusion criteria included all patients with unresectable CRLM, treated with hepatic artery infusion chemotherapy during the aforementioned time-period. Resectability was determined by a multi-disciplinary tumor board. Patients were determined to be anatomically unresectable if resection could not be performed with preservation of at least two contiguous segments or biologically unresectable if there were more than eight bilobar lesions present. The primary outcome variable was objective response rate (ORR), defined per the WHO criteria as $50 \%$ decrease in tumor burden on axial imaging (15). Secondary outcome variables included overall magnitude of response as determined by total percent decrease in tumor size of the two largest lesions visualized on cross sectional imaging, conversion to resectability, overall survival and progression free survival. Standardized mutation panel testing was performed on all liver tissue specimens that included KRAS, NRAS and BRAF.

\section{Surgical approach}

All patients who were considered for HAI pump placement diagnostic laparoscopy to rule out peritoneal metastases before HAI pump placement. Patients with oligometastatic lung disease or limited lymphadenopathy within the porta hepatis distribution were considered eligible for HAI treatment if they demonstrated prior response to systemic chemotherapy. All patients with a symptomatic or right colon primary tumor underwent synchronous resection at the time of HAI pump placement. For those with an asymptomatic rectal or rectosigmoid lesion, the primary tumor was left in situ and only HAI pump placement 
Table 1 Demographic, hospital and tumor specific data for KRAS and wild type patients

\begin{tabular}{lcc}
\hline Variable & KRAS mut & Wild-type \\
\hline Age, years, median [range] & $57.3[38-73]$ & $55.0[34-67]$ \\
Sex, male & $28 \%$ & $69 \%$ \\
Race, White or Caucasian & $91 \%$ & 0.61 \\
BMI, median [range] & $27.8[20.3-31.1]$ & 0.10 \\
Synchronous liver metastases & $82 \%$ & $27.3[21.3-40.1]$ \\
Operative duration, mins, median [range] & $250[197-474]$ & $77 \%$ \\
Length of stay, days, median [range] & $7[3-13]$ & $228[122-401]$ \\
Number of liver lesions, median [range] & $11[3-59]$ & $3[2-11]$ \\
Size of largest lesion, cm ${ }^{2}$, median [range] & $4.2[1.6-9.0]$ & $12[1-54]$ \\
Extrahepatic disease & $36 \%$ & 0.17 \\
Nr of prior systemic chemotherapy cycles, median [range] & $7.5[3-17]$ & 0.04 \\
Nr of HAl cycles, median (IQR) & $5(1.5)$ & 0.49 \\
\hline
\end{tabular}

HAI, hepatic arterial infusion; KRAS, The Kirsten rat sarcoma; BMI, body mass index.

was performed. Cholecystectomy and removal of the common hepatic artery lymph node were performed as standard parts of each procedure. Early initiation of HAI chemotherapy ( $<2$ weeks postoperatively) was standard. All patients received floxuridine $(0.12 \mathrm{mg} / \mathrm{kg} /$ day $)$ in conjunction with dexamethasone, delivered in standard 28 -day cycles. Systemic chemotherapy was resumed 4-6 weeks postoperatively and aligned with day 1 of the HAI chemotherapy cycle. Standard concomitant systemic chemotherapy consisted of a combination of leucovorin, 5 -fluorouracil and irinotecan (FOLFIRI) in most cases.

\section{Statistical analysis}

Twenty-five patients met inclusion criteria. Mean age was 59 (range, 35-77) years and 13 patients (52\%) were male. Eleven patients harbored a KRAS mutation, one patient had a BRAF V600E mutation and 13 patients were KRAS- and BRAF-wild type. Of those with a KRAS mutation, majority had codon 12 mutations (66.7\%) while two (18.2\%) patients had a codon 13 and one patient an A146T mutation. Data on demographics, tumor, and mutational status along with surgical outcomes including operative duration, length of stay, and 30-day morbidity as per the Clavien-Dindo classification were gathered (16). Long-term pump-related complications were defined as an adverse event directly related to the pump itself or pump chemotherapy. Tumor response was determined as the ratio of tumor size before HAI therapy and at the time of greatest response during or at conclusion of therapy for the two largest target lesions. Tumor size was determined on CT imaging by multiplying maximal diameter to longest perpendicular diameter in the same plane. Reassessment of resectability was done at 3 -month intervals after initiation of therapy. Patients were stratified into two groups for analysis according to their mutation status (KRAS-mut or wild-type). One patient with BRAF V600E mutation was excluded from the performed analyses. Continuous variables were compared using the student's $t$-test or Mann Whitney $U$ test depending on normality of the respective data. Categorical data were compared using the chi-square test. Survival is depicted using Kaplan-Meier curves with the log-rank test was utilized to compare survival between groups. Statistical analysis was completed using SPSS v27.0.0.0 (IBM Corp., Armonk, NY, USA).

\section{Results}

KRAS-mut and wild type patients were similar in terms of demographic, operative- and tumor characteristic (Table 1). Twelve patients (7/13 wild type, 5/11 KRAS-mut) had undergone prior resection of their primary tumor at the time of HAI pump placement. Eight patients (2/13 wild type, 5/11 KRAS-mut, 1/1 BRAF) had synchronous resection of 
Table 2 Tumor response based on mutation status

\begin{tabular}{|c|c|c|c|}
\hline Variable & KRAS mut & Wild-type & $P$ value \\
\hline ORR, n (\%) & $7(64 \%)$ & $13(100 \%)$ & 0.03 \\
\hline Conversion to resectability, $\mathrm{n}(\%)$ & $2(18 \%)$ & $8(62 \%)$ & 0.05 \\
\hline Progression free survival, months, median $(95 \% \mathrm{Cl})$ & 8.9 (95\% Cl: 4.7-13.1) & 9.8 (95\% Cl: 6.4-13.1) & 0.68 \\
\hline
\end{tabular}

ORR, objective response rate.

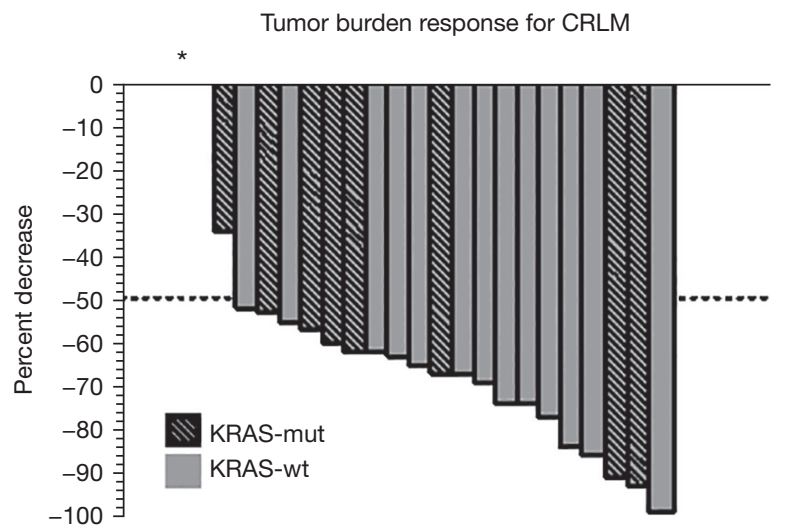

Figure 1 Waterfall plot demonstrating percentage decrease in tumor burden by mutation status. Asterix $\left(^{*}\right)$ depicts three patients (all KRAS-mut) who had disease progression while on HAI chemotherapy $(+257 \%,+30 \%$ and $+14 \%)$. HAI, hepatic arterial infusion; CRLM, colorectal cancer liver metastases.

their primary tumor at the time of HAI pump placement. All patients had undergone prior systemic chemotherapy before starting HAI therapy, of which 14 had received one prior regimen and 10 patients two prior regimens. The median number of prior chemotherapy cycles did not differ between the two groups ( 6 vs. $7.5 ; \mathrm{P}=0.76)$. The most common first line systemic chemotherapy regimen utilized was a combination of leucovorin, 5-fluorouracil and oxaliplatin (FOLFOX) with or without targeted agents (bevacizumab, cetuximab, panitumumab), $n=21$. Secondline regimens consisted of FOLFIRI in all 10 patients. The number of patients with extrahepatic disease was similar between groups (36\% vs. 31\%).

\section{Complications}

Eight patients (32\%) suffered perioperative complications of which 7 (28\%) were mild (Clavien-Dindo grade 1-2). One patient suffered a severe complication that consisted of postoperative shock of unknown etiology, requiring take-back to the operating room for an abdominal exploration which was without significant findings. This patient recovered and was eventually able to undergo HAI therapy. No 90-day mortality was witnessed. Long-term pump related complications were witnessed in four patients. One pump pocket infection occurred and was managed with prolonged antibiotics until completion of therapy and pump removal. Three other patients suffered biliary complications, either strictures or biloma formation requiring endoscopic or interventional radiology intervention. Two of those required discontinuing HAI chemotherapy infusion permanently, both after 6 cycles had been delivered.

\section{Tumor response and survival}

Median follow-up time was 14.6 months (range, 436.6 months). Overall ORR was $80 \%(20 / 25)$ and median tumor burden reduction $63.5 \%$ (range, $-257-100 \%$ ). KRAS-mut patients had a smaller median tumor reduction with HAI therapy when compared to wild type, $58 \%$ (range, $-257-94 \%$ ) vs. 70\% (range, 53-100\%) respectively; $\mathrm{P}=0.04$ (Table 2). Figure 1 shows individual response to HAI therapy for KRAS-mut and wild type patients. Three KRAS-mut patients suffered disease progression during treatment while all wild type patients had some degree of favorable response. ORR was lower for KRAS-mut patients $(7 / 11,64 \%$ vs. $13 / 13,100 \%$; $\mathrm{P}=0.03)$. KRAS-mut patients were less likely to convert to resectable status with HAI therapy $(2 / 11,18 \%$ vs. $8 / 13,62 \% ; \mathrm{P}=0.05)$. At the end of follow-up, overall survival in the KRAS-mut group was $45 \%(5 / 11)$ compared to $77 \%$ for wild type patients $(\mathrm{P}=0.30)$. Median survival was 23.7 (95\% CI: 14.3-33.1) months for the whole cohort. For KRAS-mut patients, median survival was 23.7 (95\% 


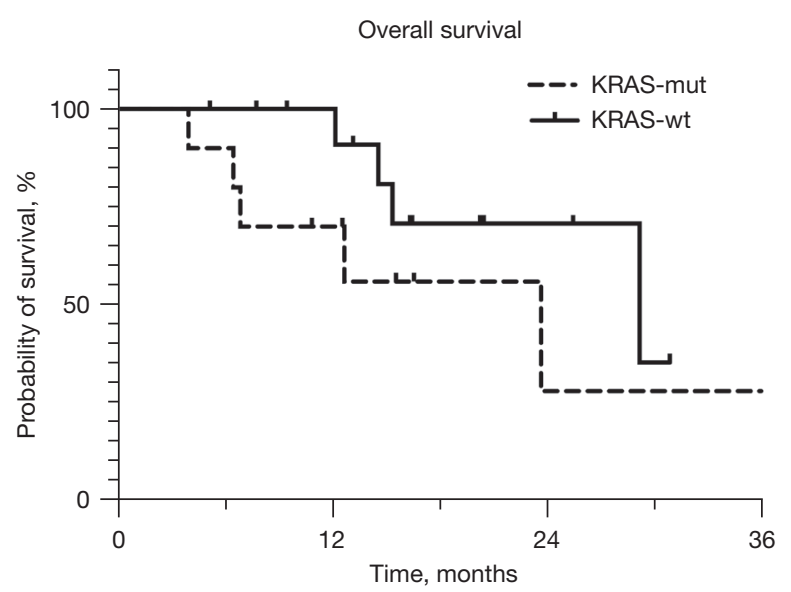

Figure 2 Kaplan-Meier survival probability for KRAS-mut and wild type patients.

CI: 2.8-44.6) months while for KRAS-wt patients median survival has not been reached. Figure 2 shows Kaplan-Meier survival probability between the two groups. Wild type and KRAS-mut groups were similar in terms of progression free survival [9.8 (95\% CI: 6.4-13.1) vs. 8.9 (95\% CI: 4.7-13.1) months], $\mathrm{P}=0.68$. There was no difference in hepatic progression free survival between KRAS-mut [20.5 (95\% CI: 6.1-34.9) months] and wild-type patients [12.7 (95\% CI: 5.6-19.8) months], $\mathrm{P}=0.63$.

\section{Discussion}

We set out to investigate whether tumor response in patients treated with hepatic artery infusion chemotherapy for unresectable CRLM is affected by KRAS mutational status. Our findings include worse ORR (64\% vs. 100\%) and smaller decrease in tumor burden (median: $58 \% \mathrm{vs}$. $70 \%$ ) for KRAS-mut patients compared to wild-type. Additionally, fewer KRAS-mut patients converted to resectable (18\% vs. $62 \%)$. These results are not surprising given that multiple reports, including a 2016 meta-analysis, have associated KRAS-mut with worse prognosis in patients undergoing hepatic resection for colorectal cancer liver metastases (17).

Nevertheless, the negative impact of KRAS mutation does not negate the benefits of HAI pump therapy. Indeed, the ORR of $64 \%$ in our KRAS-mut group is well within the $47-90 \%$ range of what other authors have described with combination HAI and systemic chemotherapy for unresectable disease $(11,18-21)$. When comparing these data to the efficacy of modern systemic chemotherapy alone it should be noted that majority of data for HAI chemotherapy comes from patients undergoing $2 \mathrm{nd}$ or $3 \mathrm{rd}$ line therapy. Although aggressive 1st line systemic regimens have produced up to $80 \%$ response rate (22), second line systemic therapies have carried more disappointing results. Studies utilizing modern 2 nd line chemotherapy regimens have described tumor response rates between 20-25\% and a median 12 month survival $(23,24)$. So far, HAI chemotherapy in conjunction with systemic chemotherapy has therefore provided superior response rates in the $2 \mathrm{nd}$ and 3 rd line setting.

Conversion to resectability in our cohort was $62 \%$ among KRAS-wt patients and $18 \%$ among KRAS-mut patients for an overall conversion rate of $42 \%$. These results are similar to previous reports from high-volume centers where the conversion rate has been $25-47 \%$ with majority of patients undergoing HAI chemotherapy as 2nd or 3 rd line treatment as well $(8,11,25)$. Scarce data exists on conversion rates with systemic therapy alone in comparable cohorts of pretreated patients. While conversion after first line therapy has been reported has high as $50 \%(26,27)$, many have criticized resectability criteria utilized by some as too strict, thus limiting meaningful conclusions. Other authors have reported a more modest $15-30 \%$ conversion to resection with modern first line chemotherapy regimens (27-29). Studies investigating 2 nd line therapies have not reported any patients converting to resectable status although a survival benefit has been noted in select cohorts $(24,30)$. This achievement of resectability may be the most profound impact of HAI therapy. Though median survival has shown modest improvements, the tails of Kaplan-Meier curves show that those resected can derive lasting benefit as seen in series out of Memorial Sloan Kettering Cancer Center (9). Median hepatic progression free survival was similar between the two groups in our cohort (12.7 vs. 20.5 months; $\mathrm{P}=0.63)$. However, limited conclusions can be made based on these findings given the small sample size of this study.

There is evidence to support that response to HAI chemotherapy correlates with timing of treatment. Some authors have pointed out dwindling efficacy in heavily pretreated patients and have advocated for earlier implementation $(19,25,31)$. D'Angelica and colleagues reported a 3 -year survival of $75 \%$ for the chemo-naïve portion of their study cohort with unresectable CRLM treated with HAI + systemic therapy, compared to $55 \%$ 3 -year survival of the whole cohort, of which $65 \%$ had received 1 or 2 prior treatments (32). These results should 
prompt further investigation into the optimal timing of HAI chemotherapy, ideally as either 1st or 2nd line therapy.

Limitations of this study include its retrospective nature, small sample size and short follow-up time, thus limiting meaningful survival analysis. Nevertheless, these results show a clear difference in treatment response based on mutational status even though excellent response rates were seen in both groups. Further prospective studies are necessary to validate these findings as well as to define the optimal timing of HAI chemotherapy in patients with unresectable CRLM. An ideal design would include prospective design with standardization of timing for HAI therapy in the setting of unresectable CRLM stratified by mutational status. Hopefully, these questions can be addressed in the newly formed national HAI consortium which includes over 20 centers.

\section{Conclusions}

KRAS mutation negatively affects tumor response to HAI chemotherapy in patients with unresectable CRLM. Nevertheless, our findings show a benefit from the treatment that is superior to what is described with systemic chemotherapy alone. Based on our findings molecular profiling for selection of patients for this treatment is not warranted, however, these results could help aid with patient discussion on expected results and prognosis.

\section{Acknowledgments}

Funding: None.

\section{Footnote}

Reporting Checklist: The authors have completed the REMARK reporting checklist. Available at https://jgo. amegroups.com/article/view/10.21037/jgo-21-514/rc

Data Sharing Statement: Available at https://jgo.amegroups. com/article/view/10.21037/jgo-21-514/dss

Peer Review File: Available at https://jgo.amegroups.com/ article/view/10.21037/jgo-21-514/prf

Conflicts of Interest: All authors have completed the ICMJE uniform disclosure form (available at https://jgo.amegroups. com/article/view/10.21037/jgo-21-514/coif). The authors have no conflicts of interest to declare.
Ethical Statement: The authors are accountable for all aspects of the work in ensuring that questions related to the accuracy or integrity of any part of the work are appropriately investigated and resolved. The study was conducted in accordance with the Declaration of Helsinki (as revised in 2013). The study was approved by the institutional review board of Spectrum Health (IRB00000883, study ID: 2020-314). Individual consent for this retrospective analysis was waived.

Open Access Statement: This is an Open Access article distributed in accordance with the Creative Commons Attribution-NonCommercial-NoDerivs 4.0 International License (CC BY-NC-ND 4.0), which permits the noncommercial replication and distribution of the article with the strict proviso that no changes or edits are made and the original work is properly cited (including links to both the formal publication through the relevant DOI and the license). See: https://creativecommons.org/licenses/by-nc-nd/4.0/.

\section{References}

1. Siegel RL, Miller KD, Jemal A. Cancer statistics, 2020. CA Cancer J Clin 2020;70:7-30.

2. Manfredi S, Lepage C, Hatem C, et al. Epidemiology and management of liver metastases from colorectal cancer. Ann Surg 2006;244:254-9.

3. Tomlinson JS, Jarnagin WR, DeMatteo RP, et al. Actual 10-year survival after resection of colorectal liver metastases defines cure. J Clin Oncol 2007;25:4575-80.

4. Adam R, Avisar E, Ariche A, et al. Five-year survival following hepatic resection after neoadjuvant therapy for nonresectable colorectal. Ann Surg Oncol 2001;8:347-53.

5. Adams RB, Aloia TA, Loyer E, et al. Selection for hepatic resection of colorectal liver metastases: expert consensus statement. HPB (Oxford) 2013;15:91-103.

6. Masi G, Loupakis F, Pollina L, et al. Long-term outcome of initially unresectable metastatic colorectal cancer patients treated with 5-fluorouracil/leucovorin, oxaliplatin, and irinotecan (FOLFOXIRI) followed by radical surgery of metastases. Ann Surg 2009;249:420-5.

7. Sullivan RD, Norcross JW, Watkins E Jr. Chemotherapy of Metastatic Liver Cancer by Prolonged Hepatic-artery Infusion. N Engl J Med 1964;270:321-7.

8. Dhir M, Jones HL, Shuai Y, et al. Hepatic Arterial Infusion in Combination with Modern Systemic Chemotherapy is Associated with Improved Survival Compared with Modern Systemic Chemotherapy Alone in Patients with 
Isolated Unresectable Colorectal Liver Metastases: A

Case-Control Study. Ann Surg Oncol 2017;24:150-8.

9. Groot Koerkamp B, Sadot E, Kemeny NE, et al. Perioperative Hepatic Arterial Infusion Pump Chemotherapy Is Associated With Longer Survival After Resection of Colorectal Liver Metastases: A Propensity Score Analysis. J Clin Oncol 2017;35:1938-44.

10. Kemeny N, Huang Y, Cohen AM, et al. Hepatic arterial infusion of chemotherapy after resection of hepatic metastases from colorectal cancer. N Engl J Med 1999;341:2039-48.

11. Kemeny NE, Melendez FD, Capanu M, et al. Conversion to resectability using hepatic artery infusion plus systemic chemotherapy for the treatment of unresectable liver metastases from colorectal carcinoma. J Clin Oncol 2009;27:3465-71.

12. Tosi F, Magni E, Amatu A, et al. Effect of KRAS and BRAF Mutations on Survival of Metastatic Colorectal Cancer After Liver Resection: A Systematic Review and Meta-Analysis. Clin Colorectal Cancer 2017;16:e153-63.

13. Phipps AI, Buchanan DD, Makar KW, et al. KRASmutation status in relation to colorectal cancer survival: the joint impact of correlated tumour markers. Br J Cancer 2013;108:1757-64.

14. Gholami S, Kemeny NE, Boucher TM, et al. Adjuvant Hepatic Artery Infusion Chemotherapy is Associated With Improved Survival Regardless of KRAS Mutation Status in Patients With Resected Colorectal Liver Metastases: A Retrospective Analysis of 674 Patients. Ann Surg 2020;272:352-6.

15. World Health Organization. Who handbook for reporting results of cancer treatment. World Health Orgiaization, 1979. Available online: https://apps.who.int/iris/ handle/10665/37200

16. Dindo D, Demartines N, Clavien PA. Classification of surgical complications: a new proposal with evaluation in a cohort of 6336 patients and results of a survey. Ann Surg 2004;240:205-13.

17. Passiglia F, Bronte G, Bazan V, et al. Can KRAS and BRAF mutations limit the benefit of liver resection in metastatic colorectal cancer patients? A systematic review and metaanalysis. Crit Rev Oncol Hematol 2016;99:150-7.

18. Kemeny NE, Niedzwiecki D, Hollis DR, et al. Hepatic arterial infusion versus systemic therapy for hepatic metastases from colorectal cancer: a randomized trial of efficacy, quality of life, and molecular markers (CALGB 9481). J Clin Oncol 2006;24:1395-403.

19. Pak LM, Kemeny NE, Capanu M, et al. Prospective phase
II trial of combination hepatic artery infusion and systemic chemotherapy for unresectable colorectal liver metastases: Long term results and curative potential. J Surg Oncol 2018;117:634-43.

20. Kemeny N, Seiter K, Niedzwiecki D, et al. A randomized trial of intrahepatic infusion of fluorodeoxyuridine with dexamethasone versus fluorodeoxyuridine alone in the treatment of metastatic colorectal cancer. Cancer 1992;69:327-34.

21. Fiorentini G, Cantore M, Rossi S, et al. Hepatic arterial chemotherapy in combination with systemic chemotherapy compared with hepatic arterial chemotherapy alone for liver metastases from colorectal cancer: results of a multicentric randomized study. In Vivo 2006;20:707-9.

22. Marques RP, Duarte GS, Sterrantino C, et al. Triplet (FOLFOXIRI) versus doublet (FOLFOX or FOLFIRI) backbone chemotherapy as first-line treatment of metastatic colorectal cancer: A systematic review and meta-analysis. Crit Rev Oncol Hematol 2017;118:54-62.

23. Fornaro L, Vasile E, Masi G, et al. Outcome of secondline treatment after first-line chemotherapy with the GONO FOLFOXIRI regimen. Clin Colorectal Cancer 2012;11:71-6.

24. Giantonio BJ, Catalano PJ, Meropol NJ, et al. Bevacizumab in combination with oxaliplatin, fluorouracil, and leucovorin (FOLFOX4) for previously treated metastatic colorectal cancer: results from the Eastern Cooperative Oncology Group Study E3200. J Clin Oncol 2007;25:1539-44.

25. Ammori JB, Kemeny NE, Fong Y, et al. Conversion to complete resection and/or ablation using hepatic artery infusional chemotherapy in patients with unresectable liver metastases from colorectal cancer: a decade of experience at a single institution. Ann Surg Oncol 2013;20:2901-7.

26. Folprecht G, Grothey A, Alberts S, et al. Neoadjuvant treatment of unresectable colorectal liver metastases: correlation between tumour response and resection rates. Ann Oncol 2005;16:1311-9.

27. Skof E, Rebersek M, Hlebanja Z, et al. Capecitabine plus Irinotecan (XELIRI regimen) compared to 5-FU/LV plus Irinotecan (FOLFIRI regimen) as neoadjuvant treatment for patients with unresectable liver-only metastases of metastatic colorectal cancer: a randomised prospective phase II trial. BMC Cancer 2009;9:120.

28. Schwartzberg LS, Rivera F, Karthaus M, et al. PEAK: a randomized, multicenter phase II study of panitumumab plus modified fluorouracil, leucovorin, and oxaliplatin (mFOLFOX6) or bevacizumab plus mFOLFOX6 in 
patients with previously untreated, unresectable, wild-type KRAS exon 2 metastatic colorectal cancer. J Clin Oncol 2014;32:2240-7.

29. Folprecht G, Gruenberger T, Bechstein WO, et al. Tumour response and secondary resectability of colorectal liver metastases following neoadjuvant chemotherapy with cetuximab: the CELIM randomised phase 2 trial. Lancet Oncol 2010;11:38-47.

30. Nielsen DL, Palshof JA, Larsen FO, et al. A systematic review of salvage therapy to patients with metastatic colorectal cancer previously treated with fluorouracil, oxaliplatin and irinotecan $+/$ - targeted therapy. Cancer

Cite this article as: Kolbeinsson HM, Preihs R, Bengel A, Chandana S, Assifi MM, Chung MH, Wright GP. Kirsten rat sarcoma (KRAS) oncogene mutation predicts magnitude of response and outcomes in hepatic arterial infusion pump therapy of unresectable colorectal liver metastases. J Gastrointest Oncol 2022;13(1):163-170. doi: 10.21037/jgo-21-514
Treat Rev 2014;40:701-15.

31. Lévi FA, Boige V, Hebbar M, et al. Conversion to resection of liver metastases from colorectal cancer with hepatic artery infusion of combined chemotherapy and systemic cetuximab in multicenter trial OPTILIV. Ann Oncol 2016;27:267-74.

32. D'Angelica MI, Correa-Gallego C, Paty PB, et al. Phase II trial of hepatic artery infusional and systemic chemotherapy for patients with unresectable hepatic metastases from colorectal cancer: conversion to resection and long-term outcomes. Ann Surg 2015;261:353-60. 